\title{
Association between cardiovascular disease risk factors and occurrence of venous thromboembolism:
}

\section{A time-dependent analysis}

Keattiyoat Wattanakit ${ }^{1}$, Pamela L. Lutsey ${ }^{2}$, Elizabeth J. Bell ${ }^{2}$, Heather Gornik ${ }^{3}$, Mary Cushman $^{4}$, Susan R. Heckbert ${ }^{5}$, Wayne D. Rosamond ${ }^{6}$, and Aaron R. Folsom ${ }^{2}$

${ }^{1}$ Cardiology, HeartCare Midwest, Peoria, Illinois, USA

2Epidemiology \& Community Health, School of Public Health, University of Minnesota, Minneapolis, Minnesota, USA

${ }^{3}$ Vascular Medicine, Cleveland Clinic, Cleveland, Ohio, USA

${ }^{4}$ Department of Medicine, University of Vermont, Colchester, Vermont, USA

${ }^{5}$ Epidemiology, University of Washington, Seattle, Washington, USA

${ }^{6}$ Department of Epidemiology, University of North Carolina, Chapel Hill, North Carolina, USA

\section{Summary}

Apart from obesity, it remains controversial whether atherosclerosis and its cardiovascular risk disease (CVD) factors are associated with risk of venous thromboembolism (VTE). Using data from the Atherosclerosis Risk in Communities study (ARIC), we evaluated associations between CVD risk factors and incident VTE in a cohort of 15,340 participants who were free a history of VTE and/or anticoagulant use on enrolment. The CVD risk factors were updated during the follow-up period. Over a mean follow-up time of 15.5 years (237,375 person-years), 468 participants had VTE events. Adjusting for demographic variables and body mass index (BMI), current smokers were at greater risk [HR of 1.44 (95\% CI: 1.12-1.86)] compared to non-smokers. There was a positive monotonic association between BMI and VTE risk. Individuals with a BMI $35 \mathrm{~kg} / \mathrm{m}^{2}$ had a HR for VTE of 3.09 (95\%CI: 2.26-4.23) compared to those with normal BMI $\left(<25 \mathrm{~kg} / \mathrm{m}^{2}\right)$. Greater physical activity was associated with lower VTE risk in a demographic adjusted model; however, this association became non-significant following adjustment for BMI. Alcohol intake, diabetes, hypertension, high-density lipoprotein and low-density lipoprotein cholesterol, and triglycerides were not associated with VTE risk. In conclusion, among the wellestablished CVD risk factors, only current smoking and obesity were independently associated with VTE risk in this large cohort where risk factors were updated serially during follow-up. This finding corroborates that the pathogenesis of venous disease differs from that of atherosclerotic disease.

\section{Keywords}

Deep-vein thrombosis; pulmonary embolism; risk factors

\section{(c) Schattauer 2012}

Correspondence to: Keattiyoat Wattanakit, MD, MPH, HeartCare Midwest - Cardiology, 5405 N Knoxville Ave, Peoria, Illinois 61614, USA, Tel.: +1 309357 3740, Fax: +1 309357 3740, topwatta@gmail.com.

Conflicts of interest

None declared. 


\section{Introduction}

Despite substantial interest, it remains unclear whether atherosclerotic cardiovascular disease (CVD) is related to risk of deep-vein thromboembolism (DVT) and pulmonary embolism (PE), collectively referred to as venous thromboembolism (VTE). In general, case-control studies have found a positive association between markers of subclinical atherosclerotic CVD and risk of VTE $(1,2)$, while prospective cohort studies reported no association $(3,4)$. It is generally believed that if an association between atherosclerotic CVD and VTE exists, the mechanism is presumed to relate to the sharing of common risk factors between the two diseases. To date, among CVD risk factors, only obesity is consistently associated with VTE risk (5-7), whereas the roles of diabetes, hypertension, smoking, total cholesterol, high density lipoprotein (HDL) and low-density lipoprotein (LDL) cholesterol, triglycerides, alcohol non-use, and physical inactivity are less clear (7-9). One possible reason for the lack of clarity is that these CVD risk factors, which are known to fluctuate over time, were typically measured only once at baseline in existing cohort studies (years before VTE events), or once after VTE cases were identified in case-control studies. It may be difficult to demonstrate associations with risk factors when, in cohort studies, VTE events occur years after the initial baseline measurements. To date, there is only one study that has analysed the association between CVD risk factors and VTE events in a time-dependent manner (6). Using data from the ARIC, we therefore conducted a study to investigate the association between CVD risk factors and VTE risk, updating risk factors during follow-up. The findings of this study should help clarify whether the contribution of risk factors to venous disease differs from that of atherosclerotic disease.

\section{Materials and methods}

\section{Study population}

The ARIC Study is a community-based, prospective study that investigates the etiology and natural history of CVD. Detailed descriptions of the study design and objectives have been published elsewhere (10). Briefly, the study enrolled 15,792 adults aged 45 to 64 years at baseline from four US communities: Forsyth County, NC; Jackson, MS; Minneapolis, MN; and Washington County, MD. Cardiovascular risk factors were collected at the baseline examination conducted between 1987 - 1989. The cohort underwent reexamination visits at roughly three-year intervals, with a 93\% return rate for visit 2 (1990-1992), 86\% for visit 3 (1993-1995), and 81\% for visit 4 (1996-1998). Informed consent was obtained from participants, with approval of methods by the institutional review boards at each study center.

\section{Measurement of cardiovascular risk factors}

At the baseline visit, the participants underwent a standardised medical history and examination that included interviews and fasting venipuncture. Participants were classified as never, former, or current alcohol drinkers. Pack-years of smoking were calculated by multiplying the average number of cigarettes per day by the number of years smoked and dividing by 20. Physical activity was assessed using the Baecke sports questionnaire, with scores ranging from 1 (low) to 5 (high), and participants were categorised as low $(<2)$ moderate (2 to 4), or high ( $\geq 4$ ) (11). Participants were asked to bring all current medications. Medication types were recorded, including cholesterol-lowering medications, beta-blockers, angiotensin-converting enzyme inhibitors, or other antihypertensive medications. Anthropometrics, including weight and height, were obtained while the participant was wearing a scrub suit. Body mass index (BMI) was calculated as weight in kilograms divided by the square of height in meters $\left(\mathrm{kg} / \mathrm{m}^{2}\right)$. 
Fasting blood samples were drawn from an antecubital vein for measurement of lipids, glucose, and chemistries. Laboratory assays for triglycerides, high-density lipoprotein (HDL) cholesterol, and calculated LDL cholesterol were performed in standardised research laboratories (12). Diabetes was defined as a fasting serum glucose level $\geq 7.0 \mathrm{mM}(126 \mathrm{mg} /$ $\mathrm{dl})$, non-fasting glucose level $\geq 11.1 \mathrm{mM}(200 \mathrm{mg} / \mathrm{dl})$, physician diagnosis of diabetes, or current use of any diabetes medication. Hypertension was defined as seated diastolic blood pressure (DBP) $\geq 90 \mathrm{mmHg}$, systolic blood pressure (SBP) $\geq 140 \mathrm{mmHg}$, or use of antihypertensive medications within the past two weeks.

\section{VTE identification and classification}

ARIC participants were followed through 2005 and were contacted annually by telephone or through clinic visits. Hospitalisations were identified by participants and by surveillance of local hospital discharge lists within ARIC sites. All records with possible VTE were identified and copied.

DVT was defined on the basis of duplex ultrasound or venogram or, in rare cases, by impedance plethysmography, computed tomography (CT), or autopsy (13). Definite PE required ventilation/perfusion scanning showing multiple segmental or subsegmental mismatched perfusion defects, or a positive pulmonary angiogram, CT, or autopsy. VTE events were further classified as unprovoked or provoked (occurring within 90 days of major trauma, surgery, hospitalisation, or marked immobility or associated with active cancer or chemotherapy).

\section{Statistical analyses}

Of 15,792 ARIC participants at baseline, we excluded, due to small numbers, 48 participants who were neither white nor African-American participants and 55 African-Americans from Washington County and Minneapolis suburbs. We further excluded 89 participants with anticoagulant use at baseline, and 260 participants who had a baseline history of VTE. The 123 participants who reported anticoagulant use (confirmed by checking medication bottles) during follow-up visits were censored at the visit prior to this use. Follow-up time was calculated as the time elapsed between the baseline visit and the first VTE event, death, date of last known contact, or December 31,2005. Less than 7\% of the cohort was lost to followup for VTE hospitalisations.

Means and counts of participant characteristics by visit were computed. Cox regression was used to calculate hazard ratios (HR) and 95\% confidence intervals (CI) of incident VTE by CVD risk factor level. Because these risk factors change over time, and more recent levels may be more highly associated with VTE risk, we modeled the risk factor variables as timedependent exposure variables using data from each of the four ARIC visits between 19871998. An analysis involving time-dependent variables uses not just the baseline values for the risk factors under consideration, but also incorporates the values at later examination visits (in this case, ARIC visits 2-4). The HRs that are estimated are essentially for the averaged value of the risk factors, rather than a single baseline value. The advantage of this analysis is that it more accurately represents the long-term level of the risk factor by accommodating changes in risk factors over time. If a participant was missing a risk factor value for a given visit, the previous visit's risk factor value was carried forward until another value was obtained.

HRs were computed with each risk factor level category modelled as an indicator variable so that there was no assumption of linearity. Linear trends across the categories were calculated by including the risk factor categories in the model as a continuous variable. A test for interaction for each risk factor with $\log ($ time $)$ showed that the proportional hazards 
assumption was not violated. Model 1 adjusted for age (continuous), race (white, African American), field center (Forsyth County, Jackson, Minneapolis, Washington County), and sex (male, female). Model 2 additionally adjusted for BMI (continuous) as a time-varying covariate. Multiplicative interactions of each CVD risk factor and age $(<55$ years, $>55$ years at baseline) were also tested using cross-product terms.

Blood pressure and lipids could change over time due to use of anti-hypertensive and lipid lowering medications. Therefore, we accounted for medication use by including as timevarying covariates the measured baseline SBP, DBP, and lipid values together with use of anti-hypertensive (yes, no) and lipid lowering (yes, no) medications. As a secondary analysis, for those on anti-hypertensive medications a constant of 15 was added to the measured blood pressure values (14). For those on lipid lowering medications, constants (depending on specific type of medications) were added to the original LDL and HDL cholesterol and triglycerides (15).

\section{Results}

Among 15,340 participants at the first visit, the mean age was 54 years. Almost $27 \%$ were African-Americans, and $45 \%$ were men. 468 participants had VTE events over the mean follow-up time of 15.5 years (237,375 person-years). Table 1 shows risk factor levels at each visit. Compared to baseline, the percentage of participants who reported being active (in quartile 4 ) increased (19.9\% at visit 1 vs. $23.3 \%$ at visit 3 ). The percentage of participants who used tobacco or alcohol decreased $(26.2 \%$ at visit 1 vs. $15.0 \%$ at visit 4 for current tobacco use and $14.2 \%$ at visit 1 vs. $11.3 \%$ at visit 4 for alcohol use $\geq 100 \mathrm{~g} / \mathrm{weeks})$. The prevalence of obesity and diabetes increased over the years. At visit 1,9\% of participants had BMI $235 \mathrm{~kg} / \mathrm{m}^{2}$ and $12 \%$ had diabetes. At visit 4 , the prevalence had increased to $12.2 \%$ and $16.7 \%$, respectively. The prevalence of hypertension also increased from $34.9 \%$ at visit 1 to $47.1 \%$ at visit 4 . On the other hand, LDL cholesterol declined (26.0\% with LDL $\geq 160$ at visit 1 vs. $12.6 \%$ with LDL $\geq 160$ at visit 4 ) over the years, as treatment increased.

Table 2 shows HRs for incident VTE (provoked and unprovoked) by risk factor levels. In the demographic adjusted model, a higher level of physical activity was associated with a lower risk of incident VTE. This association lost statistical significance after adjustment for BMI. After adjustment for demographic variables and BMI, current smoking was associated with a $44 \%$ greater risk of VTE than non-smoking. There was a linear association between BMI and VTE risk $\left(p_{\text {trend }}=<0.0001\right.$ ). Participants with a BMI $\geq 35 \mathrm{~kg} / \mathrm{m}^{2}$ were at three-fold increased risk of VTE compared to those with a normal BMI $\left(<25 \mathrm{~kg} / \mathrm{m}^{2}\right)$. This association remained statistically significant when separately analysed for unprovoked and provoked VTE. Alcohol intake was unrelated to risk of VTE events.

Of the physiologic characteristics, in the demographic-adjusted model diabetes was associated with greater risk of incident VTE, while high HDL cholesterol and high LDL cholesterol were associated with lower risk. Triglycerides, blood pressure, and use of statins were not associated with risk of VTE. After additional adjustment for BMI, there seemed to be a trend toward a lower VTE risk with higher LDL level, while no other associations were observed between physiologic characteristics and risk of VTE. Results were similar in secondary analyses when we added constants to account for the antihyperlipidaemic and antihypertensive medications (data not shown). In addition, there were no interactions of age with all of the CVD risk factors with p-value $<0.05$ after accounting for multiple testing. 


\section{Discussion}

Using a time-dependent analysis, we found that current smoking and obesity were the only atherosclerotic CVD risk factors associated with risk of VTE. Physical activity was inversely associated with risk of VTE, but was confounded by obesity. Alcohol use, diabetes, hypertension, or lipid levels were not associated with VTE risk. The same findings were observed for unprovoked and provoked VTE.

The findings of our study are in contradiction to a published meta-analysis (8). In that study, obesity, hypertension, diabetes mellitus, triglycerides, and low HDL were risk factors for VTE in unadjusted analyses, raising the possibility that risk factor modifications may help reduce VTE risk. However, the meta-analysis was based on pooling both case-control and cohort studies with different populations and inclusion criteria. Furthermore, a major limitation of this meta-analysis is that the analyses did not adjust for important confounders such as age and obesity, which are known to associate with both CVD risk factors and VTE. Hence, this particular issue makes interpretation of this meta-analysis very difficult as reported positive findings could be due to confounding.

Whether CVD risk factors increase VTE risk has been a focus of many cohort and casecontrol studies. Most studies (6,7,16-19), including ours, agree that current smoking is associated with increased VTE risk. A Danish study found a dose-response relationship between smoking and VTE risk. The relative risk for VTE (both unprovoked and provoked VTE) was 2.6 (95\%CI: $1.5-4.7)$ for those currently smoking > 25 cigarettes per day compared to non-smokers (17). Biologically, smoking's association with VTE may be mediated through hypercoagulability and impaired fibrinolysis (20). Hence, smoking cessation may not only decrease the risk of arterial atherosclerosis, but also VTE. However, some studies failed to show an association of smoking with VTE $(5,21-23)$. In a prior LITE publication (5), which included 19,293 men and women from the ARIC and CHS studies, Tsai et al. reported that former or current smokers were not at higher risk for VTE compared to non-smokers. The discrepancy between that analysis and our current analysis may be explained by the fact that the Tsai analysis had a shorter follow-up time (median 8 years vs. 15.5 years in our study), fewer events, and included participants $>65$ years from the CHS study.

Our findings corroborate well documented findings that obesity is an important VTE risk factor $(5-7,16,18,24,25)$. Existing literature on the relation between physical activity and VTE risk has been inconclusive, with some studies reporting that physical activity is associated with lower VTE risk (26), others showing that physical activity is associated with higher VTE risk $(27,28)$, and others reporting no association $(5,6)$. In this analysis, physical inactivity was associated with a higher risk of incident VTE, but this was attenuated after accounting for BMI. Obesity likely resides on the causal pathway between physical inactivity and VTE, therefore the observed attenuation of the association with adjustment for BMI is not surprising.

The roles of diabetes, hypertension, and dyslipidemia in the pathogenesis of VTE risk are controversial. The LITE study by Tsai et al. previously reported that diabetes was a modest risk factor for VTE (5), whereas our findings did not corroborate this. Other studies reported that diabetes did not increase VTE risk $(6,16,29,30)$ or that the association was confounded by other VTE risk factors such as major surgery, medical illness, or residence in a nursing home (31).

Our analyses also showed no relation between hypertension and risk of incident VTE. 
Consistent with our findings, the Copenhagen Heart Study reported no association between SBP and VTE (6). Based on a self-reported questionnaire, however, the Nurses' Health Study, which followed 112,822 women 30 to 55 years of age over 16 years, reported that hypertension, after multivariable adjustment (including obesity), was a modest risk factor for VTE, with HR $=1.5$ (95\%CI: $1.2-2.0)(16)$.

Few studies specifically examined the association of lipid levels and VTE. In this analysis, after accounting for BMI, high LDL level appeared to be associated with lower VTE risk. The explanation for this finding is unclear and is perhaps related to chance. Levels of HDL cholesterol and triglycerides, however, were unrelated to VTE risk. Another previous LITE analysis reported that HDL cholesterol, subfractions of HDL cholesterol (HDL-2 and HDL3), and apolipoprotein A-I were not associated with VTE (32). The Copenhagen Heart Study also reported no association of dyslipidaemia with VTE (6). In contrast, a small casecontrol study suggested that men $<55$ years with VTE had low levels of HDL and elevated levels of LDL compared to matched controls (33). Lipoprotein subclass analyses showed that these differences reflected lower levels of large HDL particles and higher levels of small LDL particles.

Although we unexpectedly observed an inverse association between LDL level and VTE risk, statin treatment when evaluated independently (Table 2) was in the direction of lower VTE risk, but was not statistically significant. The JUPITER trial (34) and a post-trial analysis of the Heart and Estrogen/progestin Replacement Therapy Study (HERS) (35) showed a reduction in VTE risk with statin therapy. The beneficial effects of statins are thought to be mediated through reduction of thrombin generation and increased activity of the protein $\mathrm{C}$ anticoagulant pathway $(20,36)$, not the reduction of cholesterol levels. It is possible that we failed to observe a significant association between statins and VTE risk due to lack of statistical power, as there were relatively few events among statin users.

In several instances prior studies reported associations between diabetes, hypertension, and dyslipidaemia and risk of VTE, while in this re-analysis of the LITE data we did not. It is possible that the studies reporting positive associations may have overestimated the risk due to residual confounding, and perhaps by obesity. These studies also usually measured the risk factors only once at baseline. In our study, we adjusted for changes in clinical characteristics and use of medications.

Strengths of our study are that this is a large, well-designed epidemiologic study. Measurements of CVD risk factors and validation of VTE events were standardised. The large sample size of the study should have provided sufficient power to detect associations of CVD risk factors on VTE risk. Furthermore, participants were examined and covariates were adjusted and updated during the follow-up time prior to onset of VTE. We also acknowledge limitations. Given that many participants had multiple CVD risk factors, it is conceivable that some may have died from competing risk such as arterial events prior to the development of VTE. In addition, VTE cases would have been missed if VTE events were not hospitalised or recognised by clinicians. These limitations are true of many epidemiologic studies and would typically lead to bias toward finding no association between risk factors and VTE. Lastly, although the follow-up period ended in 2005, data on CVD risk factors were available and updated serially between 1987-1998.

Among the well-established risk factors for atherosclerotic CVD, only smoking and obesity were independently associated with VTE risk in this study. This finding corroborates that the pathogenesis of venous disease is different from that of atherosclerotic disease.

Avoidance of smoking and obesity may help prevent both venous and arterial diseases. 


\section{Acknowledgments}

The Atherosclerosis Risk in Communities Study is carried out as collaborative study supported by National Heart, Lung, and Blood Institute contracts (HHSN268201100005C, HHSN268201100006C, HHSN268201100007C, HHSN268201100008C, HHSN 268201100009C, HHSN2682011000010C, HHSN2682011000011C, HHSN2682011000012C). The authors thank the staff and participants of the ARIC study for their important contributions.

\section{References}

1. Prandoni P, Bilora F, Marchiori A, et al. An association between atherosclerosis and venous thrombosis. N Engl J Med. 2003; 348:1435-1441. [PubMed: 12686699]

2. Hong C, Zhu F, Du D, et al. Coronary artery calcification and risk factors for atherosclerosis in patients with venous thromboembolism. Atherosclerosis. 2005; 183:169-174. [PubMed: 15939424]

3. van der Hagen PB, Folsom AR, Jenny NS, et al. Subclinical atherosclerosis and the risk of future venous thrombosis in the Cardiovascular Health Study. J Thromb Haemost. 2006; 4:1903-1908. [PubMed: 16961598]

4. Reich LM, Folsom AR, Key NS, et al. Prospective study of subclinical atherosclerosis as a risk factor for venous thromboembolism. J Thromb Haemost. 2006; 4:1909-1913. [PubMed: 16836659]

5. Tsai AW, Cushman M, Rosamond WD, et al. Cardiovascular risk factors and venous thromboembolism incidence: the longitudinal investigation of thromboembolism etiology. Arch Intern Med. 2002; 162:1182-1189. [PubMed: 12020191]

6. Hoist AG, Jensen G, Prescott E. Risk factors for venous thromboembolism: results from the Copenhagen City Heart Study. Circulation. 2010; 121:1896-1903. [PubMed: 20404252]

7. Lutsey PL, Virnig BA, Durham SB, et al. Correlates and consequences of venous thromboembolism; The Iowa Women's Health Study. Am J Public Health. 2010; 100:1506-1513. [PubMed: 19910349]

8. Ageno W, Becattini C, Brighton T, Selby R, Kamphuisen PW. Cardiovascular risk factors and venous thromboembolism: a meta-analysis. Circulation. 2008; 117:93-102. [PubMed: 18086925]

9. Mahmoodi BK, Gansevoort RT, Veeger NJ, et al. Microalbuminuria and risk of venous thromboembolism. J Am Med Assoc. 2009; 301:1790-1797.

10. The ARIC investigators. The Atherosclerosis Risk in Communities (ARIC) Study: design and objectives. Am J Epidemiol. 1989; 129:687-702. [PubMed: 2646917]

11. Baecke JA, Burema J, Frijters JE. A short questionnaire for the measurement of habitual physical activity in epidemiological studies. Am J Clin Nutr. 1982; 36:936-942. [PubMed: 7137077]

12. Friedewald WT, Levy RI, Fredrickson DS. Estimation of the concentration of low-density lipoprotein cholesterol in plasma, without use of the preparative ultracentrifuge. Clin Chem. 1972; 18:499-502. [PubMed: 4337382]

13. Cushman M, Tsai AW, White RH, et al. Deep vein thrombosis and pulmonary embolism in two cohorts: the longitudinal investigation of thromboembolism etiology. Am J Med. 2004; 117:1925. [PubMed: 15210384]

14. Tobin MD, Sheehan NA, Scurrah KJ, et al. Adjusting for treatment effects in studies of quantitative traits: antihypertensive therapy and systolic blood pressure. Stat Med. 2005; 24:29112935. [PubMed: 16152135]

15. Lutsey PL, Pankow R-TL, Alonso JSA, et al. Relation of lipid gene scores to longitudinal trends in lipid levels and incidence of abnormal lipid levels among Caucasians: The Atherosclerosis Risk in Communities (ARIC) study. Circ Cardiovasc Genet. 2012; 5:73-80. [PubMed: 22057756]

16. Goldhaber SZ, Grodstein F, Stampfer MJ, et al. A prospective study of risk factors for pulmonary embolism in women. J Am Med Assoc. 1997; 277:642-645.

17. Severinsen MT, Kristensen SR, Johnsen SP, et al. Smoking and venous thromboembolism: a Danish follow-up study. J Thromb Haemost. 2009; 7:1297-1303. [PubMed: 19566546]

18. Hansson PO, Eriksson H, Welin L, et al. Smoking and abdominal obesity: risk factors for venous thromboembolism among middle-aged men: "the study of men born in 1913". Arch Intern Med. 1999; 159:1886-1890. [PubMed: 10493318] 
19. Pomp ER, Rosendaal FR, Doggen CJ. Smoking increases the risk of venous thrombosis and acts synergistically with oral contraceptive use. Am J Hematol. 2008; 83:97-102. [PubMed: 17726684]

20. Lee KW, Lip GY. Effects of lifestyle on hemostasis, fibrinolysis, and platelet reactivity: a systematic review. Arch Intern Med. 2003; 163:2368-2392. [PubMed: 14581258]

21. Goldhaber SZ, Savage DD, Garrison RJ, et al. Risk factors for pulmonary embolism. The Framingham Study. Am J Med. 1983; 74:1023-1028. [PubMed: 6859053]

22. Daly E, Vessey MP, Hawkins MM, et al. Risk of venous thromboembolism in users of hormone replacement therapy. Lancet. 1996; 348:977-980. [PubMed: 8855852]

23. Samama MM. An epidemiologic study of risk factors for deep vein thrombosis in medical outpatients: the Sirius study. Arch Intern Med. 2000; 160:3415-3420. [PubMed: 11112234]

24. Stein PD, Beemath A, Olson RE. Obesity as a risk factor in venous thromboembolism. AmJ Med. 2005; 118:978-980. [PubMed: 16164883]

25. Severinsen MT, Kristensen SR, Johnsen SP, et al. Anthropometry, body fat, and venous thromboembolism: a Danish follow-up study. Circulation. 2009; 120:1850-1857. [PubMed: 19858417]

26. van Stralen KJ, Le Cessie S, Rosendaal FR, et al. Regular sports activities decrease the risk of venous thrombosis. J Thromb Haemost. 2007; 5:2186-2192. [PubMed: 17697136]

27. Glynn RJ, Rosner B. Comparison of risk factors for the competing risks of coronary heart disease, stroke, and venous thromboembolism. Am J Epidemiol. 2005; 162:975-982. [PubMed: 16207808]

28. van Stralen KJ, Doggen CJ, Lumley T, et al. The relationship between exercise and risk of venous thrombosis in elderly people. J Am Geriatr Soc. 2008; 56:517-522. [PubMed: 18179500]

29. Cogo A, Bernardi E, Prandoni P, et al. Acquired risk factors for deep-vein thrombosis in symptomatic outpatients. Arch Intern Med. 1994; 154:164-168. [PubMed: 8285811]

30. Ageno W, Prandoni P, Romualdi E, et al. The metabolic syndrome and the risk of venous thrombosis: a case-control study. J Thromb Haemost. 2006; 4:1914-1918. [PubMed: 16848878]

31. Heit JA, Leibson CL, Ashrani AA, et al. Is diabetes mellitus an independent risk factor for venous thromboembolism? : a population-based case-control study. Arterioscler Thromb Vase Biol. 2009; 29:1399-1405.

32. Chamberlain AM, Folsom AR, Heckbert SR, et al. High-density lipoprotein cholesterol and venous thromboembolism in the Longitudinal Investigation of Thromboembolism Etiology (LITE). Blood. 2008; 112:2675-2680. [PubMed: 18614761]

33. Deguchi H, Pecheniuk NM, Elias DJ, et al. High-density lipoprotein deficiency and dyslipoproteinemia associated with venous thrombosis in men. Circulation. 2005; 112:893-899. [PubMed: 16087810]

34. Glynn RJ, Danielson E, Fonseca FA, et al. A randomized trial of rosuvastatin in the prevention of venous thromboembolism. N Engl J Med. 2009; 360:1851-1861. [PubMed: 19329822]

35. Grady D, Wenger NK, Herrington D, et al. Postmenopausal hormone therapy increases risk for venous thromboembolic disease. The Heart and Estrogen/progestin Replacement Study. Ann Intern Med. 2000; 132:689-696. [PubMed: 10787361]

36. Undas A, Brummel-Ziedins KE, Mann KG. Statins and blood coagulation. Arterioscler Thromb Vase Biol. 2005; 25:287-294. 


\section{What is known about this topic?}

- It is unclear whether cardiovascular disease risk factors increase risk of venous thromboembolism (VTE).

- Prior studies consistently reported that obesity is associated with VTE. However, the associations between other cardiovascular disease risk factors and VTE are inconsistent.

- One criticism for inconsistent finding is that these cardiovascular disease risk factors, which are known to fluctuate over time, are usually measured only once at baseline when VTE events occurred many years after the initial measurements. This makes it difficult to demonstrate associations.

\section{What does this paper add?}

- This study analyses the associations between cardiovascular disease risk factors and VTE risk by serially updating risk factors and adjusting for use of antihypertensive and cholesterol lowering medications during follow-up time.

- The results should help clarify the associations. 
Table 1

Participant characteristics by visit, ARIC 1987-1998 (1).

\begin{tabular}{|c|c|c|c|c|}
\hline Visit number & V1 (1987-1989) & V2 (1990-1992) & V3 (1993-1995) & V4 (1996-1998) \\
\hline $\mathrm{N}$ & 15340 & 13867 & 12368 & 11068 \\
\hline \multicolumn{5}{|l|}{ Demographics } \\
\hline Age, years (SD) & $54.1(5.8)$ & $57.0(5.7)$ & $59.9(5.7)$ & $62.7(5.7)$ \\
\hline African-Americans, n (\%) & $4114(26.8)$ & $3430(24.7)$ & $2856(23.1)$ & $2503(22.6)$ \\
\hline Male, n (\%) & $6905(45.0)$ & $6210(44.8)$ & $5486(44.4)$ & $4867(44.0)$ \\
\hline \multicolumn{5}{|l|}{ Behaviours } \\
\hline \multicolumn{5}{|l|}{ Physical activity, $\mathrm{n}(\%)^{2}$} \\
\hline Q1 & $4343(28.4)$ & - & $3046(24.8)$ & - \\
\hline Q2 & $3600(23.6)$ & - & $2685(21.9)$ & - \\
\hline Q3 & $4297(28.1)$ & - & $3676(30.0)$ & - \\
\hline Q4 & 3035 (19.9) & - & $2864(23.3)$ & - \\
\hline HRT use among women, $\mathrm{n}(\%)^{3}$ & $1654(19.9)$ & $2252(27.0)$ & $2779(33.4)$ & $2758(33.4)$ \\
\hline \multicolumn{5}{|l|}{ Smoking, n (\%) } \\
\hline Never & $6379(41.6)$ & $5487(39.7)$ & $5065(41.1)$ & $4564(41.7)$ \\
\hline Former & $4926(32.1)$ & $5245(37.9)$ & $5057(41.0)$ & $4745(43.3)$ \\
\hline Current & $4020(26.2)$ & $3103(22.4)$ & $2207(17.9)$ & $1647(15.0)$ \\
\hline \multicolumn{5}{|l|}{ Alcohol intake in g/week, $\mathrm{n}(\%)$} \\
\hline 0 & $9340(61.3)$ & $9155(65.9)$ & $7774(63.6)$ & $7435(67.9)$ \\
\hline $1-99$ & $3737(24.5)$ & $3027(21.9)$ & $2896(23.7)$ & $2277(20.8)$ \\
\hline$\geq 100$ & $2159(14.2)$ & $1686(12.2)$ & $1561(12.8)$ & $1237(11.3)$ \\
\hline \multicolumn{5}{|l|}{ BMI, $\mathrm{kg} / \mathrm{m}^{2}, \mathrm{n}(\%)$} \\
\hline$<25$ & $5092(33.3)$ & $4327(31.3)$ & $3424(27.7)$ & $2823(25.6)$ \\
\hline $25-29$ & $6031(39.4)$ & 5527 (39.9) & $4863(39.4)$ & $4391(39.8)$ \\
\hline $30-34$ & $2819(18.4)$ & $2651(19.2)$ & $2634(21.3)$ & $2471(22.4)$ \\
\hline 235 & $1373(9.0)$ & $1334(9.6)$ & $1429(11.6)$ & $1346(12.2)$ \\
\hline \multicolumn{5}{|l|}{ Physiologic characteristics } \\
\hline \multicolumn{5}{|l|}{ Diabetes status, n (\%) } \\
\hline Normal & $7742(50.9)$ & $5112(37.0)$ & $5692(48.4)$ & $5115(46.8)$ \\
\hline IFG & $5649(37.1)$ & $6602(47.8)$ & $4447(36.1)$ & $3995(36.5)$ \\
\hline Prevalent diabetes & $1829(12.0)$ & $2090(15.1)$ & $1898(15.4)$ & $1826(16.7)$ \\
\hline \multicolumn{5}{|l|}{$\mathrm{BP}, \mathrm{mmHg}, \mathrm{n}(\%)$} \\
\hline \multicolumn{5}{|l|}{ Systolic } \\
\hline$<120$ & $7873(51.4)$ & $7111(51.3)$ & $5394(43.6)$ & $4006(36.2)$ \\
\hline $120-139$ & $5112(33.4)$ & $4580(33.0)$ & $4574(37.0)$ & $4399(39.8)$ \\
\hline$\geq 140$ & $2341(15.3)$ & $2171(15.7)$ & $2394(19.4)$ & $2648(24.0)$ \\
\hline \multicolumn{5}{|l|}{ Diastolic } \\
\hline$<80$ & $11106(72.5)$ & $10834(78.2)$ & $9659(78.1)$ & 8905 (80.6) \\
\hline
\end{tabular}




\begin{tabular}{|c|c|c|c|c|}
\hline Visit number & V1 (1987-1989) & V2 (1990-1992) & V3 (1993-1995) & V4 (1996-1998) \\
\hline $80-89$ & $3037(19.8)$ & $2283(16.5)$ & $2088(16.9)$ & $1690(15.3)$ \\
\hline$\searrow 0$ & $1183(7.7)$ & $746(5.4)$ & $615(5.0)$ & $458(4.1)$ \\
\hline HTN, n (\%) & $5319(34.9 \%)$ & $4951(35.8 \%)$ & $5009(40.7 \%)$ & $5193(47.1 \%)$ \\
\hline BP med, n(\%) & $4638(30.3)$ & 4489 (32.4) & $4589(37.1)$ & $4735(42.8)$ \\
\hline \multicolumn{5}{|l|}{ Lipid values, mg/dl, n (\%) } \\
\hline \multicolumn{5}{|l|}{ HDL cholesterol } \\
\hline$<40$ & $4025(26.7)$ & 4067 (29.6) & $3039(24.7)$ & $3070(28.0)$ \\
\hline $40-59$ & $7087(46.9)$ & $6437(46.8)$ & $5806(47.2)$ & 5217 (47.6) \\
\hline 260 & 3989 (26.4) & 3239 (23.6) & $3470(28.2)$ & $2685(24.5)$ \\
\hline \multicolumn{5}{|l|}{ LDL cholesterol } \\
\hline$<100$ & $2382(16.0)$ & $2285(16.9)$ & $2564(21.2)$ & $2651(24.6)$ \\
\hline $100-129$ & $4225(28.4)$ & $4306(31.8)$ & 4077 (33.6) & $3933(36.4)$ \\
\hline $130-159$ & 4406 (29.6) & $4069(30.0)$ & $3525(29.1)$ & $2856(26.5)$ \\
\hline$\geq 160$ & $3866(26.0)$ & $2887(21.3)$ & $1959(16.2)$ & $1356(12.6)$ \\
\hline \multicolumn{5}{|l|}{ Triglycerides } \\
\hline$<150$ & $10904(72.2)$ & $9653(70.0)$ & $8161(66.3)$ & $7189(65.5)$ \\
\hline $150-199$ & $2157(14.3)$ & $2080(15.1)$ & $2130(17.3)$ & $1855(16.9)$ \\
\hline$\geq 200$ & $2041(13.5)$ & $2050(14.9)$ & $2024(16.4)$ & $1928(17.6)$ \\
\hline Lipid lowering medication, $\mathrm{n}(\%)$ & $431(2.8)$ & $860(6.2)$ & $1155(9.4)$ & $1539(14.0)$ \\
\hline Statin use, n (\%) & $81(0.5)$ & $322(2.3)$ & $668(5.4)$ & $1215(11.0)$ \\
\hline
\end{tabular}

1 Participants who reported a history of VTE and/or anticoagulant use at baseline were excluded. Participants who reported anticoagulant use at follow-up were excluded at the visit before use was reported. Values are mean (SD) or $\mathrm{n}(\%)$.

2 Only measured at visit 1 and visit 3 .

3 Only measured at visit 1. ARIC, Atherosclerosis Risk in Communities; BMI, body mass index; HRT, hormone replacement therapy; Q, quartile; $\mathrm{SD}$, standard deviation; V, visit; HTN, hypertension; BP, blood pressure. 


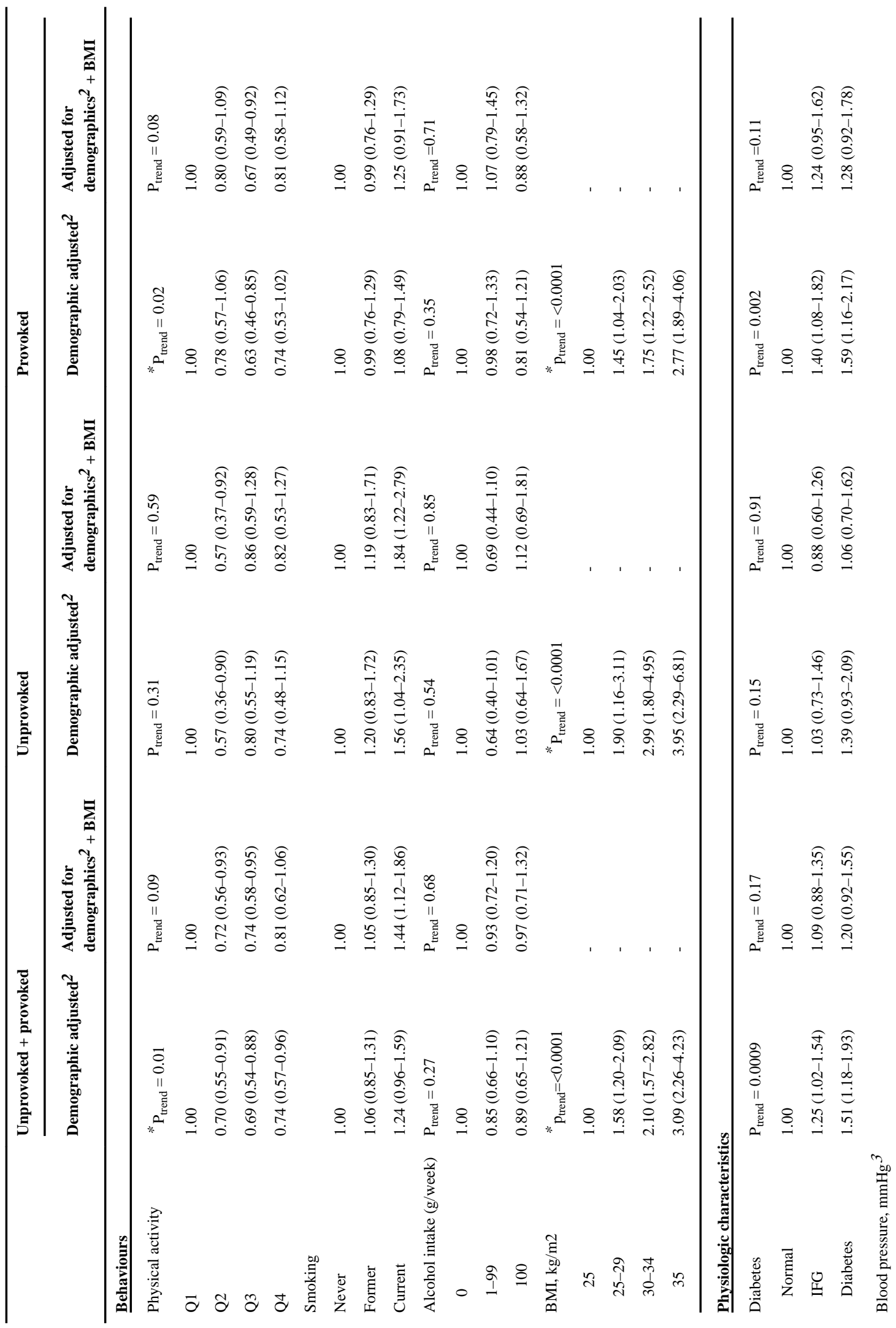




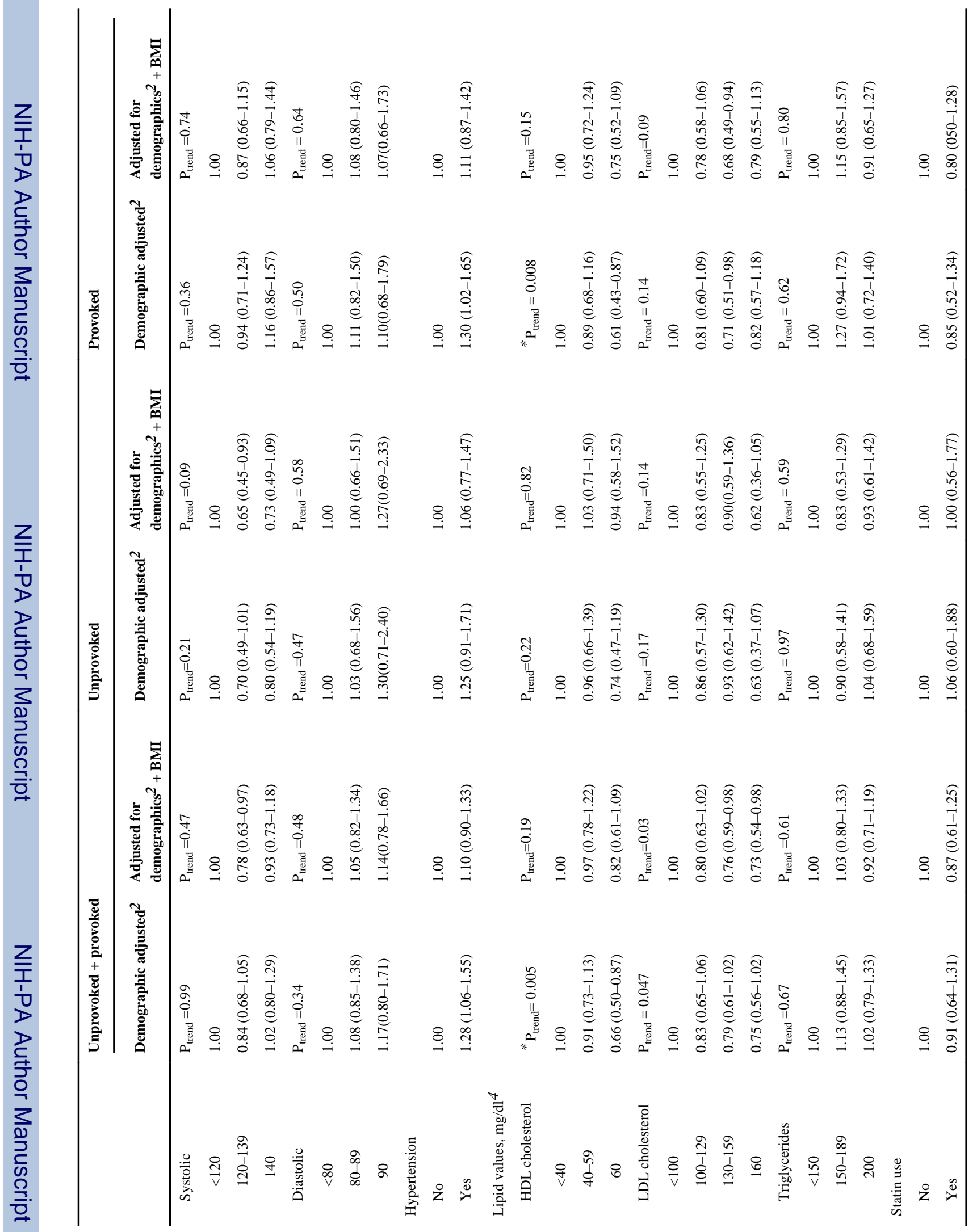




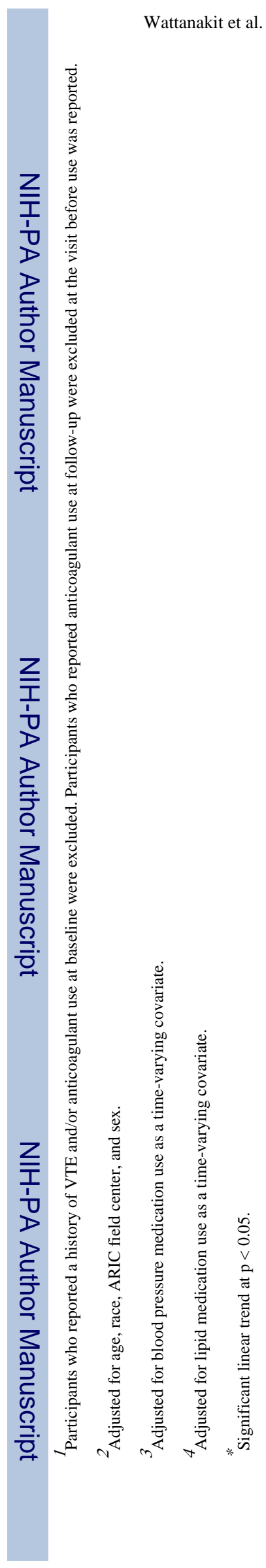

Page 14

Thromb Haemost. Author manuscript; available in PMC 2013 September 04. 